REVIEW

\title{
Gene targeted therapeutics for liver disease in alpha-I antitrypsin deficiency
}

\author{
Caitriona McLean* \\ Catherine M Greene* \\ Noel G McElvaney \\ Respiratory Research Division, Dept. \\ Medicine, Royal College of Surgeons \\ in Ireland, Education and Research \\ Centre, Beaumont Hospital, Dublin \\ 9, Ireland; *Each of these authors \\ contributed equally to this work
}

\begin{abstract}
Alpha-1 antitrypsin (A1AT) is a $52 \mathrm{kDa}$ serine protease inhibitor that is synthesized in and secreted from the liver. Although it is present in all tissues in the body the present consensus is that its main role is to inhibit neutrophil elastase in the lung. A1AT deficiency occurs due to mutations of the A1AT gene that reduce serum A1AT levels to $<35 \%$ of normal. The most clinically significant form of A1AT deficiency is caused by the Z mutation (Glu342Lys). ZA1AT polymerizes in the endoplasmic reticulum of liver cells and the resulting accumulation of the mutant protein can lead to liver disease, while the reduction in circulating A1AT can result in lung disease including early onset emphysema. There is currently no available treatment for the liver disease other than transplantation and therapies for the lung manifestations of the disease remain limited. Gene therapy is an evolving field which may be of use as a treatment for A1AT deficiency. As the liver disease associated with A1AT deficiency may represent a gain of function possible gene therapies for this condition include the use of ribozymes, peptide nucleic acids (PNAs) and RNA interference (RNAi), which by decreasing the amount of aberrant protein in cells may impact on the pathogenesis of the condition.
\end{abstract}

Keywords: alpha-1 antitrypsin deficiency, siRNA, peptide nucleic acid, ribozymes

\section{Introduction}

Alpha-1 antitrypsin (A1AT) is an acute phase $52 \mathrm{kDa} 418$ amino acid glycoprotein that is primarily synthesized and secreted by the hepatocytes in the liver ${ }^{1}$ though it is also actively transcribed and secreted in smaller amounts by cells including neutrophils, mononuclear phagocytes, enterocytes, ${ }^{2}$ and human respiratory epithelial cells. ${ }^{3}$

It is present in all tissues of the body with its primary role being to inhibit the enzyme neutrophil elastase (NE). ${ }^{4}$ It also inhibits to a much lesser degree trypsin, chymotrypsin, cathepsin $\mathrm{G}$, plasmin, thrombin, tissue kallikrein, factor $\mathrm{Xa}$, plasminogen, and proteinase 3 (Table 1$)^{5}$

A1AT has other pathobiologically relevant functions in addition to elastase inhibition. There is a direct pro-survival effect of A1AT through inhibition of lung alveolar endothelial cell apoptosis. Primary pulmonary endothelial cells internalize human A1AT which co-localizes with and inhibits staurosporine-induced caspase-3 activation. A1AT is among several serum proteins that can rescue serum withdrawal-induced apoptosis. ${ }^{6}$ Inhibition of structural alveolar cell apoptosis by A1 AT represents a novel protective mechanism of this serpin against emphysema. ${ }^{6}$

A1AT has also been reported to play an immunoregulatory role, to inhibit neutrophil superoxide production, to induce the release of macrophage-derived interleukin-1 (IL-1) receptor agonist, and to increase hepatocyte growth factor production in human lung fibroblasts in vitro. In vivo A1AT has been shown to protect against tumor necrosis factor- $\alpha(\mathrm{TNF} \alpha)$ or endotoxin-induced lethality and in a mouse model of lung inflammation and connective tissue breakdown. It was shown that A1AT binds to the secreted enteropathogenic Escherichia coli proteins EspB and EspD thereby 
Table I The AIAT alleles and their effects

\begin{tabular}{|c|c|c|c|}
\hline Allele & Genetic change & $\begin{array}{l}\text { Serum A I AT levels } \mu M \\
\text { (Less than I I = deficient) }\end{array}$ & Effects \\
\hline M & Normal & Homozygous (MM): 20-48 & Normal \\
\hline S & Glu246Val & Homozygous (SS): I5-33 & $\begin{array}{l}\text { - Mild plasma deficiency } \\
\text { - Not clinically significant usually } \\
\text { - No hepatocellular aggregation }\end{array}$ \\
\hline Z & Glu342Lys & $\begin{array}{l}\text { Heterozygous (MZ): } 12-35 \text { Heterozygous (SZ): } \\
\text { 8-19 Homozygous (ZZ): } 2.5-7\end{array}$ & $\begin{array}{l}\text { Carrier } \\
\text { Deficient } \\
\text { Most significant deficiency } \\
\text { - Associated with emphysema and liver disease } \\
\text { - Intra-hepatocellular polymerization key feature }\end{array}$ \\
\hline $\mathrm{S}_{\text {iiyana }}$ & Ser53Phe & & $\begin{array}{l}\text { - Severe plasma deficiency } \\
\text { - Intra-hepatocellular polymerization }\end{array}$ \\
\hline$M_{\text {malton }}$ & $\Delta$ Phe52 & & $\begin{array}{l}\text { - Severe plasma deficiency } \\
\text { - Intra-hepatocellular polymerization } \\
\text { - Next to } Z \text { allele, most frequently associated with } \\
\text { liver disease }\end{array}$ \\
\hline I & Arg39Cys & & $\begin{array}{l}\text { - Rare } \\
\text { - Mild plasma deficiency } \\
\text { - Intra-hepatocellular polymerization }\end{array}$ \\
\hline Null & & Homozygous: 0 & $\begin{array}{l}\text { - Complete plasma deficiency } \\
\text { - No polymerization } \\
\text { - Not associated with liver disease }\end{array}$ \\
\hline
\end{tabular}

reducing their hemolysis of red blood cells. Thus A1AT may not only afford protection against proteolytic injury, but may also have the potential to neutralize microbial activities and to exert effects on the regulation of innate immunity. In this regard there is growing evidence that A1AT is also able to inhibit lipopolysaccharides (LPS)-induced inflammatory responses in vitro and in vivo. ${ }^{7}$

A1AT is a member of the serine proteinase inhibitor (Serpin) family of proteins. This family also includes $\alpha-1$ antichymotrypsin, $\alpha-2$ antiplasmin, plasminogen activator I, thyroxine-binding globulin and angiotensin, among others. Serpins are single chain proteins containing a conserved domain structure of 370-390 residues, usually flanked by amino or carboxyl terminal extensions. The serpins exist in a metastable state and their energy is released by cleavage of their reactive centre. A target protease binds to and cleaves the reactive centre of the serpin which then transposes the protease to the other end of the serpin molecule. In the process the protease is crushed against the serpin, with a loss of structural integrity that ensures its destruction. ${ }^{8}$

A crucial factor in the destruction is the strength of the bond between serpin and protease. ${ }^{8}$ Inhibitory serpins interact with their target proteinase at the reactive site which is located within a loop structure 30-40 amino acids from the carboxyl terminus. ${ }^{9}$
The specificity of a serpin for its protease is determined by the side chain of the amino acid in the reactive centre of the serpin. The mature A1AT protein consists of a single chain of amino acids with three $\mathrm{N}$-asparaginyl-linked complex carbohydrate side chains. ${ }^{5}$ As already stated the target protein of A1AT is NE. A1AT has a methionine at its reactive centre which enables it to inhibit $\mathrm{NE}$ as $\mathrm{NE}$ cleaves at methionyl residues. ${ }^{8}$ The predominant feature of cleaved, inactive serpins is the presence of an anti-parallel six-stranded $\beta$-sheet (the A sheet) in which the central strand contains several residues upstream from the cleavage site. ${ }^{9}$

With 80 million neutrophils from the pulmonary arterial circulation travelling through human lungs every second, the pulmonary parenchymal stroma is exposed to an array of proteolytic enzymes including proteinase-3, cathespin $\mathrm{G}$ and NE. Of these NE is thought to be the most important in the development of pulmonary emphysema. It is a $29 \mathrm{kDa}$, 220 amino acid, single chain polypeptide glycoprotein synthesized in promyelocytes and packaged in neutrophil azurophilic granules. NE is released in large quantities in response to neutrophil activation ${ }^{10}$ and is one of the few human enzymes capable of cleaving insoluble, cross-linked elastin which modulates the elastic recoil of tissues, including the alveolar walls of the lower respiratory tract. ${ }^{5}$ After an 
inflammatory event, in normal circumstances, the NE is inhibited and controlled by A1AT.

\section{Genetics of AI AT deficiency}

The A1AT gene is located on the q arm of chromosome 14 at position $14 q 32.1 .^{11,12}$ It is a 12.2-kilobase pair gene composed of seven exons and six introns. The protein is encoded by the protease inhibitor (Pi) locus. The Pi locus is highly polymorphic, resulting in different A1AT isotypes that can be detected by electrophoresis. ${ }^{13}$

There are many normal A1AT alleles, and at least four common ' $\mathrm{M}$ ' alleles. The many different alleles can be either normal, null (express no A1AT), dysfunctional (nonfunctional A1AT is expressed in the serum) or deficient (serum A1AT levels $<35 \%$ of normal). More than 100 different allelic variations of A1AT have been described. Over $95 \%$ of the population has one of the four $\mathrm{M}$ alleles. ${ }^{14}$

The numerous A1AT phenotypes are named according to their migration characteristics in a $\mathrm{pH} 4$ to $\mathrm{pH} 5$ isoelectric focusing (IEF) polyacrylamide gel. Variants displaying a higher isoelectric point are conferred with letters from the beginning of the alphabet whereas those with lower isoelectric points are named from the end of the alphabet. ${ }^{10}$ When two alleles have an identical IEF pattern and the sequence difference is known, the relevant residue is specifically indicated. For example; the two most common A1AT alleles, M1 ( $\left.\mathrm{Val}^{213}\right)$ and M1 (Ala $\left.{ }^{213}\right)$, have IEF patterns of M1, but differ at residue 213 by the neutral amino acids Val and Ala. Some rare alleles are labeled by a letter indicating the IEF position together with the birth site of the allele $\left(\mathrm{M}_{\text {procida }}\right)$. The null alleles are labeled "null" together with the place of origin ( Null $\left._{\text {bellingham }}\right) .^{5}$

One in 10 people of European descent are carriers of one of the two most common base-substitution mutations of A1AT that result in its deficiency. The more common is the $\mathrm{S}$ mutation (Glu264Val). In homozygotes this results in a $40 \%$ decrease in plasma A1AT concentrations. The most clinically significant mutation is the $\mathrm{Z}$ allele (Glu342Lys). Approximately $4 \%$ of Northern Europeans and 3\% of people in the United Sates are heterozygous for the $\mathrm{Z}$ allele (PiMZ), with approximately 1 in 2000 being homozygotes (PiZZ). Homozygosity for the $Z$ allele reduces plasma levels of A1AT to $10 \%-15 \%$ of normal. In PiZZ homozygotes, or those that co-inherit the $Z$ allele and the $\mathrm{S}$ mutation plasma concentrations of A1AT will be insufficient to ensure lifetime protection of the lungs from proteolytic damage, particularly in smokers. ${ }^{8}$

More than $90 \%$ of clinical cases of A1AT deficiency are caused by the PiZZ mutation. This occurs at an allelic frequency of 0.01 to 0.02 in North American white persons and 0.02 to 0.03 in Northern Europeans. The allelic frequency is lower in Southern Europeans (0.009) and African Americans (0.004). This demographic distribution also refers to the rarer clinically significant alleles. The null allele occurs with an allelic frequency of 0.00017 . The reason for the predominance of the $Z$ allele is unknown, but does raise the possibility that heterozygosity confers a survival advantage on the host. ${ }^{10}$

Gene-mapping studies show that the PiZ allele probably arose in northern Europe and age estimates of the A1AT variants suggest that it first appeared 107 to 135 generations ago and could have been spread in Neolithic times. The PiS deficiency allele is older. It is estimated to have arisen 279-470 generations ago and from its high incidence on the Iberia peninsula it has been suggested that PiS could have arisen in this region. ${ }^{15}$

A study of genetic epidemiologic studies from peerreviewed literature was used to establish the worldwide racial and ethnic distribution of A1AT deficiency. The database estimated that the deficiencies would be present in a variety of populations and races. ${ }^{16}$ A study of 96 Filipino infants with a history of jaundice since two weeks old, however, found an extremely low incidence of A1AT deficiency with only one patient positive for the PiS allele and negative for the periodic acid-Schiff(PAS) positive hepatic globules characteristic of the deficiency. ${ }^{17}$ A similar study of 58 children with either chronic liver disease or neonatal cholestasis syndrome was carried out in India. None of the children had A1AT-deficient phenotypes. ${ }^{18}$

Low plasma concentrations are also associated with two other rare variants of A1AT, $\mathrm{S}_{\text {iiyama }}$ (Ser53Phe) and $\mathrm{M}_{\text {malton }}$ $(\Delta \mathrm{Phe} 52)$, the commonest causes of A1AT deficiency in Japan and Sardinia, respectively. ${ }^{8} \mathrm{~S}_{\text {iiyama }}$ is also associated with PAS-positive, diastase-resistant inclusion bodies in the liver. The serine at position 53 is thought to contribute to the organization of the internal core element of the A1AT molecule. The deficient state in $\mathrm{S}_{\text {iiyama }}$ may therefore be due to the change from an uncharged polar to a nonpolar amino acid imposed on the conserved serpin backbone which may exert severe effects on the integrity of the molecule and hence alter the intracellular processing of A1AT. ${ }^{19}$

ZA1AT is the mutation which causes the most severe and most clinically relevant form of A1AT deficiency. The Z mutation (Glu342Lys) in A1AT occurs at the head of the fifth strand of the A-sheet and affects the mobility of the reactive centre loop and therefore the physical properties of A1AT. The mutation results in a unique molecular interaction 
between the reactive centre loop of one molecule and the gap in the A-sheet of another. This process can continue until large polymers form. This loop-sheet polymerisation of ZA1AT occurs spontaneously at $37{ }^{\circ} \mathrm{C} .{ }^{20}$

The loop-sheet polymerization has provided an excellent explanation for the aggregation of the Z-variant of A1AT in the liver endoplasmic reticulum (ER). The polymers of ZA1AT form aggregates. These aggregates are present as diastase-resistant PAS positive inclusions of A1AT in the ER of periportal cells. These inclusions result in a deficiency of circulating plasma A1AT, reducing plasma levels of the protein to $10 \%-15 \%$ of normal. ${ }^{21}$

Electron microscopy of the hepatocytic inclusions in PiZZ homozygotes reveals bead-like necklaces of A1AT polymers. These polymers are resistant to the usual degradative processes of the ER because the individual molecules retain their ordered structure and hence fail to elicit the chaperone binding needed to activate degradation. ${ }^{8}$ The oligosaccharide structure of the accumulated material from the PAS-positive diastase-resistant inclusions is compatible with a blockage of processing prior to entry to the Golgi. The protein accumulation occurs after core glycosylation in the endoplasmic reticulum but before the high mannose pruning which takes place in the Golgi. On extraction from the inclusions the A1AT is functionally active although this ZA1AT had only $15 \%$ of the activity of MA1AT. ${ }^{22}$ The $\mathrm{Z}$ mutation reduces the association rate between A1AT and NE by approximately fivefold. ${ }^{20}$ Therefore not only is there less A1AT in PiZZ individuals, but the population of ZA1AT molecules is less competent as an inhibitor of NE than MA1AT, and this suggests that PiZZ individuals have far less functional antielastase protection than the reduced concentration of A1AT alone would imply. ${ }^{23}$

Polymerization of A1AT and consequent globular deposits are enhanced by high temperatures. Storage is speeded up also by the increase in synthesis of A1AT because polymerization is a concentration-dependent phenomenon. Consequently hepatocytic storage accelerates during febrile illness and infectious diseases (the acute-phase response) that involve either the liver or other organs. In fact in these conditions A1AT synthesis is incremental as the body temperature rises. ${ }^{24}$ A1AT is an acute-phase reactant as its plasma levels increase during the host response to inflammation/tissue injury. ${ }^{25}$ It should be noted however that differing results have been reported regarding the effects of temperature on the secretion/polymerization of ZA1AT and it is unlikely that there is a simple relationship between febrile episodes and phenotypic expression of liver disease in A1AT-deficient patients.
The process of intra-hepatic polymerization also underlies the mild plasma deficiency of the common S (Glu264Val) and rare I (Arg39Cys) alleles of antitrypsin and the severe plasma deficiency of the $\mathrm{S}_{\text {iiyama }}$ and $\mathrm{M}_{\text {malton }}$ alleles. ${ }^{26}$

There is a strong genotype-phenotype correlation in these diseases that can be explained by the molecular instability caused by the mutation and, in particular, the rate at which the mutant forms polymers. Those mutants that cause the most rapid polymerisation cause the most retention of A1AT within the liver. This correlates with the greatest risk of liver damage and cirrhosis, and the most severe plasma deficiency. ${ }^{27}$

There is prolonged hepatic retention of the A1AT Saar allele even though this allele does not have polymerogenic properties. The retention of this allele in the ER may be even more prolonged than that of ZA1AT even though A1AT Saar does not form insoluble polymers. This indicates that there could be mechanisms other than polymerization that determine whether mutant A1AT molecules are retained in the ER. It also raises the question of whether the polymerization of ZA1AT is the cause or the result of ER retention of the protein. ${ }^{25,28}$

\section{Clinical manifestations of AI AT deficiency in the lung}

A1AT deficiency was first described by Laurell and Eriksson in $1963,{ }^{29}$ and it is associated with a substantially increased risk for the development of pulmonary emphysema by the 4 th or 5 th decade of life. ${ }^{20}$ It is thought that the emphysema is caused by the lack of fully active A1AT in the pulmonary interstitium which results in the unopposed action of proteases, the gradual destruction of pulmonary connective tissues and the loss of alveolar units. ${ }^{10}$ It appears that patients with lower rates of secretion and low plasma A1AT levels risk rapid development of emphysema whereas those with somewhat higher rates of synthesis have better protection of alveolar tissue.

Cigarette smoking markedly accelerates the development of emphysema in individuals with A1AT deficiency. This can be explained in part because the key $\mathrm{Met}^{358}$ in the active site of A1AT can be easily oxidized. When oxidation occurs the association rate constant of A1AT for NE is reduced 2000-fold. Cigarette smoke and inflammatory cells in the lower respiratory tract can oxidize the Met efficiently. ${ }^{5}$ Studies from this laboratory have demonstrated that in addition to $\mathrm{Met}^{358}$, $\mathrm{Met}^{351}$ is also susceptible to oxidation and sitedirected mutants of A1AT with alanines substituted for these key methionines are resistant to oxidative inactivation. ${ }^{30}$ 
Major differences in pathology exist between emphysema in PiMM and PiZZ individuals. This is due to the proteolytic attack and tissue destruction present in A1AT deficiency, and the fact that in its native form ZA1AT is fivefold less efficient at inhibiting neutrophil elastase than normal MA1AT. A1AT is also locally produced on the epithelial surface of the lung. This ZA1AT polymerizes at body temperature and is both an ineffective antiprotease inhibitor and a neutrophil chemoattractant. ${ }^{31}$ There is already an excess of neutrophils in the airways of individuals suffering from ZA1AT-related emphysema and the polymers cause an influx of neutrophils. The excess of neutrophils is likely due to elevated pro-inflammatory cytokines such as IL-8 and leukotriene $\mathrm{B}_{4}$. The clearance of the A1AT polymers is impaired as they are bound to the alveolar wall. This enables them to act as a chronic stimulus for neutrophil influx into ZA1AT lungs. Thus the single amino acid substitution converts an anti-inflammatory molecule into a pro-inflammatory stimulus and this contributes in part to the excessive inflammation that underlies the progressive emphysema associated with ZA1AT deficiency. ${ }^{32}$

Bronchial secretions of chronic obstructive pulmonary disease (COPD) patients with A1AT deficiency were observed to have increased levels of IL-8 and, in particular leukotriene $\mathrm{B}_{4}\left(\mathrm{LTB}_{4}\right)$. LTB 4 and IL-8 are both potent chemotactic agents capable of promoting neutrophil transendothelial migration. The absolute contribution of $\mathrm{LTB}_{4}$ and IL-8 was significantly higher in the sputum from patients with A1AT deficiency than in patients without A1AT deficiency, although the remaining sputum chemotactic activity (not accounted for by $\mathrm{LTB}_{4}$ or IL-8) did not differ significantly between the two groups. ${ }^{33} \mathrm{~A}$ possible explanation for the presence of these two chemoattractants in the sputum is the presence of free elastase as elastase has been shown to stimulate $\mathrm{LTB}_{4}$ release from alveolar macrophages as the macrophages bind uninhibited NE due to the presence of surface receptors for NE. ${ }^{34}$

Sputum inflammation and chemotactic activity can be influenced by variations in patient characteristics such as the degree of lung function impairment, the presence of acute exacerbations, cigarette smoking, corticosteroid treatment and high bacterial load..$^{33}$

\section{Clinical manifestations of A I AT deficiency in the liver}

A1AT deficiency is also associated with liver disease. The $\mathrm{PiZ}$ and $\mathrm{PiM}_{\text {malton }}$ mutations are those most frequently associated with liver disease. Sharp and colleagues (1969) first described cirrhosis in A1AT deficiency in 10 children from 6 families and later reported the characteristic intrahepatocyte PAS diastase-resistant inclusions. ${ }^{35}$

Of PiZ adults who have been systematically followed, it appears that $\sim 50 \%$ die at a mean age of $52 \mathrm{yr}$ of severe lung disease. Those subjects have mild or no signs of liver disease. Mortality of the remaining $50 \%$ occurs $\sim 10$ yr later with signs of chronic progressive liver disease at death and milder lung disease. Among PiMZ heterozygotes, lung disease is not significantly increased but chronic progressive liver disease has been found occasionally with progression to cirrhosis and, rarely, hepatoma. A German study looking at liver carcinoma and tumor-bearing tissue found that there is an increased risk of primary liver carcinoma among PiZ heterozygotes and that PiZ-associated carcinoma may develop in noncirrhotic tissue. ${ }^{36}$ However a more recent study in Florida found that there was no association between the PiMZ state and chronic liver disease in general, but among patients with liver disease there was a significantly higher number of PiMZ patients suffering from severe liver disease compared to those suffering with less severe liver disease. It was also discovered that there was a significant association between the PiMZ heterozygous state and the increased severity of liver disease and the need for transplantation in patients with hepatitis $\mathrm{C}$ virus $(\mathrm{HCV})$. These represent interim results of a long-term study into the role of heterozygous A1AT deficiency in chronic liver disease ${ }^{37}$

While homozygous inheritance of the $\mathrm{Z}$ deficiency allele is associated with an increased risk for both emphysema and liver disease, the homozygous inheritance of a null allele is associated with a higher risk of emphysema but no risk of liver disease. ${ }^{5}$ Patients with a higher rate of synthesis of ZA1AT tend to have a greater prevalence of liver disease than patients who have a low rate of expression. These second group tend towards emphysema. A1AT deficiency is the most common genetic cause of liver disease in children and predisposes adults to chronic liver disease and hepatocellular carcinoma. ${ }^{25}$

Large scale screening for A1AT deficiency in Sweden between 1972 and 1974 showed that of 200,000 neonates there were 120 PiZZ individuals, only 14 of whom had prolonged jaundice with 9 developing severe liver disease. In addition $2 \mathrm{PiZ}-, 54 \mathrm{PiSZ}$ and $1 \mathrm{PiS}-$ children were identified. ${ }^{38}$

The consistent occurrence of overt liver disease in newborn infants, in contrast to its only occasional occurrence in young adults, may be explained by the fact that the liver cells in the infants are less capable of degrading the polymerized protein. ${ }^{8}$ 
While the intrahepatocytic globules of A1AT are nontoxic in themselves, being present in all PiZ individuals including those who never develop liver disease, the accumulation does hamper the natural defenses of hepatocytes through an engorgement of the cellular synthetic pathway. This leads to a toxic gain-of-function. The accumulated ZA1AT is subjected to degradation by the ER-associated degradation pathway (ERAD).$^{39}$ The proteasome also plays an important role in the ER degradation of ZA1AT in many cell types. ${ }^{40}$ Autophagy represents a nonproteasomal mechanism by which the protein is degraded..$^{41}$ Where the protein is not degraded at a high enough rate the ER stress response is activated. The three main functional components of the ER stress response are the ER overload response (EOR), the unfolded protein response (UPR) and apoptosis. ${ }^{14}$ Endoplasmic reticulum stress responses have evolved to be protective, however when they are ineffective, toxic damage occurs demonstrating how these responses can be described as a double edged sword. ${ }^{42}$ Interestingly human hereditary chromatosis, a genetic liver disease associated with iron overload is also characterized by ER stress. ${ }^{43}$

This derangement of cellular functions renders hepatocytes more prone to damage by external insults which can have particularly damaging effects on the neonatal liver due to its relatively immature structure and function. ${ }^{24}$ In early childhood the most common presentation of A1AT deficiency's effect on the liver is prolonged jaundice. Abnormal liver function is largely self-limiting, but it can sometimes persist into adolescence. It is estimated that $5 \%-10 \%$ of A1AT deficient patients over the age of 50 will develop cirrhosis. A study examining adult patients with A1AT deficiency and chronic liver disease revealed a late onset of symptomatic hepatic abnormalities. $68 \%$ of the patients studied were 60 years or older when the liver disease was discovered. There seemed to be a later onset of the liver disease in the heterozygotes. At the time of diagnosis, the hepatic condition usually was advanced. ${ }^{44}$

The liver damage occurs through a gain-of-function mechanism, unlike the lung disease, which is due to lossof-function. The retention of the mutant ZA1AT molecules in the ER triggers a series of events that are eventually hepatotoxic. ${ }^{45}$ This gain-of-function mechanism is illustrated in ZA1AT transgenic mice. The liver disease is apparent in the mice even though normal levels of antielastases expressed by endogenous genes are still present, therefore the liver injury can not be attributed to loss of function. ${ }^{46}$

The variability in the severity of liver disease and the age of onset among patients with A1AT deficiency may be explained in part by individual variations in episodes of inflammation and hence to increased synthesis of A1AT. ${ }^{8}$ A study that involved the transfection of human skin fibroblasts with ZA1AT from patients with and without liver disease found that there was a marked delay in the degradation of the accumulated protein in the cells from those subjects with liver disease, compared to those without liver disease. Human skin fibroblasts do not express the endogenous A1AT gene, but presumably express genes involved in the processing of secretory proteins. This result would suggest that there are other genetic traits that affect the fate of the ZA1AT molecule within cells. ${ }^{45}$ Virusinduced liver disease in deficient subjects frequently assumes a chronic course because of the deranged immunologic system of these subjects. Studies have demonstrated that PiZ subjects with liver disease have anomalies of immunomodulation and anomalous activation of the complement cascade. ${ }^{24}$

Cirrhosis is favored over restitution as the proteases released by leukocytes during the inflammatory response to the hepatocytic necrosis cannot be properly counteracted and collagen and reticulum fibers are improperly rearranged during the healing process. Increased numbers of collagen bundles have been observed in the space of Disse and around hepatocytes in A1AT-deficient patients. An insufficient defense against reactive oxygen metabolites has been hypothesized to perpetuate the liver damage. ${ }^{24}$

The accumulation of ZA1AT can cause liver damage in transgenic PiZZ mice where the degree of liver damage correlates with the amount of ZA1AT accumulated in the liver of the different pedigrees of mice. Although $40 \%$ of the PiZZ mice tested were seropositive for mouse hepatitis virus (MHV), comparison of the PiZZ mice with and without MHV showed no difference in liver damage. The degree of liver damage was therefore not influenced by the MHV seropositivity; rather, it was related only to the presence of accumulated ZA1AT protein. ${ }^{46}$

Male gender and obesity have been found to predispose A1AT deficient individuals to liver disease. ${ }^{47}$ The association of the hepatitis virus with A1 AT deficiency is less clear. An Austrian study of A1AT deficient patients with chronic liver disease found that $62 \%$ of those with cirrhosis were HCV positive and $33 \%$ showed evidence of hepatitis B virus (HBV) infection. ${ }^{48}$ Another study found that the odds of having a heterozygous $\mathrm{Z}$ phenotype increased in patients with $\mathrm{HCV}$ compared to a control population, where patients with hepatitis $\mathrm{C}$ or $\mathrm{B}$ virus were 3.6 times more likely to have a heterozygous $\mathrm{Z}$ phenotype than a normal phenotype. ${ }^{49}$

Some studies, however, have found no association between hepatitis $\mathrm{C}$ infection and A1AT deficiency. ${ }^{50}$ 
A study into the PiMZ population found that the prevalence of A1AT PiMZ was no greater in hepatitis C patients than in the general population. ${ }^{51}$

The nonsteroidal anti-inflammatory drug (NSAID) indomethacin causes an increase of liver damage in transgenic ZA1AT expressing mice. It is estimated that NSAIDs are used by 15-20 million people in the United States on a long-term basis. The administration of indomethacin increases hepatic injury as indicated by the increase in hepatocellular proliferation and increased activation of caspase-9. Indomethacininduced injury is associated with activation of IL-6-STAT3 signaling, increased expression of A1AT mRNA and greater accumulation of polymerized ZA1AT in the liver. This indicates that environmental factors such as exogenous medication administration can significantly potentiate the liver injury associated with ZA1AT hepatic accumulation; NSAIDs may be especially injurious to patients with A1AT deficiency, possibly by increasing the expression and accumulation of the hepatotoxic mutant protein. ${ }^{52}$ As the $\mathrm{Z}$ mutation may lead to worse liver injury in the setting of other liver diseases interplay of cholestasis and the PiZZ phenotype was investigated in transgenic mice. A bile duct ligation (BDL) was carried out on mice possessing a transgenic alpha1-ATZ mutation and littermate controls. PiZ transgenic mice undergoing BDL developed more liver fibrosis than wild-type mice after BDL. Increased apoptosis was also noted in PiZ BDL mice. ${ }^{53}$ This shows that PiZ transgenic mice are more susceptible to liver fibrosis induced by cholestasis from BDL. The $\mathrm{Z}$ mutation of A1AT may act as a modifier gene in patients with concurrent liver diseases such as cystic fibrosis and cholestasis. Similarly the co-existence of the $\mathrm{Z}$ mutation and C282Y HFE (the protein involved in human hereditary hemochromatosis) act as potential disease modifiers with respect to each other in terms of expression on ER stress responses. ${ }^{43}$

PiZZ subjects with liver disease are prone to develop a hepatocellular type of carcinoma. Mild or severe hepatocytic dysplasia is not an infrequent sign found in biopsy specimens. Usually liver cancer develops in a patient with cirrhosis or other chronic liver disease. Occasionally however, PiZZ patients with hepatocellular carcinoma do not have cirrhosis. Usually globules are situated in the periportal hepatocytes, but in the cirrhotic nodules they are spread throughout. ${ }^{24}$

A1AT deficiency may also affect other human proteins. A recent case report involved a patient presenting with polycythemia (a known paraneopaetic manifestation of hepatoma which requires the presence of alpha-fetopro [AFP]) but with normal serum levels of AFP. This patient was found to have A1AT deficiency. Infants with neonatal hepatitis and A1AT deficiency do not have raised AFP. It was postulated that this is because A1AT is a rate limiting factor in the production of AFP. The authors raised the possibility of A1AT deficiency resulting in normal AFP in adults with hepatocellular carcinoma. ${ }^{54}$

Overall 10\% of PiZZ neonates develop hepatitis and cholestasis. Cholestasis usually occurs in the first two months, though it may persist for up to eight months. ${ }^{55}$ In early childhood patients may present with jaundice in which the stools generally contain no yellow or green pigment, indicating cholestasis and mimicking biliary atresia. All patients have hepatomegaly and about 50\% also have splenomegaly. Approximately $5 \%$ of the patients present with an increased bleeding tendency. This is due to vitamin $\mathrm{K}$ deficiency caused by the cholestasis-induced malabsorption. Less commonly children present later in childhood with hepatosplenomegaly or with cirrhosis. ${ }^{13}$

\section{Current therapeutics}

The only corrective therapy for A1AT deficient patients with severe liver disease currently is liver transplantation. The most recent study shows that the 1-, 3-, and 5-year patient survival is $89 \%, 85 \%$, and $83 \%$, respectively, for adults after transplantation versus $92 \%, 90 \%$, and $90 \%$ for pediatric patients after transplantation. ${ }^{56}$ In cases where transplantation is not possible there are palliative benefits of portocaval shunting. The beneficial effects of both breast feeding and vitamin E supplementation in cholestatic infants have been suggested. ${ }^{10}$

In 2003 the American Thoracic Society published their first new set of guidelines for the management of obstructive lung disease associated with A1AT deficiency since 1989. They recommended standard treatments as per nonhereditary emphysema.

Augmentation therapy has been given for the treatment of lung disease associated with A1AT deficiency since 1987. The levels of A1AT in serum, bronchoalveolar lavage fluid and the pulmonary interstitium, required to provide protection against proteolytic lung destruction, have been estimated from those observed in PiSZ persons who have lower than normal levels of A1AT but no demonstrable increase in premature lung disease. These levels can be reached and maintained in PiZZ and null individuals by intermittent infusions of plasma-purified A1AT. ${ }^{10}$

\section{Gene therapeutics}

As emphasized by the ATS in 2003 there is no therapy available other than liver transplantation for individuals with 
advanced A1AT-deficiency liver disease. To address this challenge there have been a number of investigations into alternative methods to treat the liver disease. As the adverse effects of A1AT deficiency are due to the expression of a single gene, the specific knockdown of this gene has high potential for the treatment of the deficiency. Gene therapy may be a possibility for treatment of the liver disease. Gene therapy was originally designed as a means to provide therapeutic merit by introducing genetic material (DNA or RNA) encoding a protein that is missing or defective into a patient's cells or tissues. Newer gene therapy approaches aimed at inhibiting the expression of a target mutant gene include RNA interference (RNAi) mediated through small interfering RNA (siRNA), peptide nucleic acids, and ribozymes. These approaches can be used to stop the production of the mutant $\mathrm{Z}$ protein, hence stopping the accumulation of the protein in the liver and providing protection against liver disease.

A recent study investigated the effects of small DNA fragments (SDFs) on A1AT. This is the DNA technology of small fragment homologous replacement (SFHR) which is versatile in that sequences can be directly altered, inserted or deleted. Treatment involves direct conversion of the mutant sequence to a wild-type genotype, thereby restoring the normal phenotype. Small DNA fragments are introduced into the cell and recombine with the genomic DNA at a targeted site, thereby producing a specific change in the sequence. SDFs including normal $M$ and ZA1AT sequences were generated and transfected into peripheral blood monocytes from PiM subjects and PiZ subjects. The defective gene could be corrected in ZA1AT monocytes in vitro with SDFs, and treatment was associated with an increase in A1AT secretion. The development of this methodology to repair the gene defect in hepatocytes could have beneficial effects on secretion, thereby protecting both the lung and liver. ${ }^{57}$

\section{RNAi and siRNA}

Post-transcriptional gene silencing (PTGS) was first observed in petunias at the end of the 1980s. Attempts to enhance the hue of petals via the introduction of a chimeric gene unexpectedly resulted in the production of completely white petals with corresponding knockdown in mRNA levels. The developmental timing of mRNA expression of the endogenous gene was not altered by introduction of an exogenous gene, but the level of the mRNA produced by the endogenous gene was seen to decrease 50-fold from wild-type levels. Somatic reversion of plants with white flowers to phenotypically parental violet flowers was associated with a coordinate rise in the steady state levels of the mRNAs produced by both the endogenous and introduced genes. ${ }^{58}$ This led to the discovery of RNA interference (RNAi).

RNA interference (RNAi) is the evolutionary-conserved process of sequence-specific, post-transcriptional gene silencing in animals and plants, initiated by double-stranded RNA (dsRNA) that is homologous in sequence to the silenced gene. ${ }^{59}$ RNAi is exclusively found in eukaryotes. This is because in prokaryotes RNase III, which is a very potent and fast ds-specific RNase, degrades dsRNA substrates as short as 12 bp. ${ }^{60}$

Although the pathway of RNAi is under constant investigation, many of the underlying mechanisms have been clarified. The process is divided into distinct steps (Figure 1). The initiation of post transcriptional gene silencing occurs on processing of a dsRNA by an enzyme known as Dicer into $\sim 22$ nt guide sequences. These guide sequences are known as small interfering RNA (siRNA). The siRNA then forms part of a multi-component nuclease, the RNA-induced silencing complex (RISC), and instructs it to destroy specific mRNAs. ${ }^{61}$ Fractionation indicates that RISC and the process generating siRNAs from dsRNA are separate, though the two enzymes responsible may interact at some point during the silencing process. RNA III family members are among the few nucleases that show specificity for dsRNA. ${ }^{62}$ Dicer is evolutionary conserved in worms, flies, plants, fungi, and mammals. ${ }^{63}$

In actively proliferating cells siRNA activity lasts for up to 1 week, whereas in terminally differentiated cells such as neurons, the silencing can last for up to 3 weeks. ${ }^{64}$ This transiency is due to the fact that mammalian cells lack the RNA-dependent RNA polymerases that amplify siRNAs in nonmammalian cells such as Caenorhabditis elegans. As a result the siRNA duplexes become progressively diluted as cells divide. ${ }^{65}$ This could be an ideal therapeutic window for many chronic diseases, for although it would necessitate repeat administrations of siRNA every few weeks this would be a short enough term to lessen concerns regarding the longterm toxicity of permanently silencing a gene, while still a long enough treatment time to be a worthwhile therapy. ${ }^{66}$ This would be particularly useful for the liver disease associated with A1AT deficiency.

The delivery in vivo of siRNAs by liposomes has proven successful. The complexation of a siRNA targeting the Bcl-2 oncogene with liposomes resulted in the successful inhibition of tumor formation in mice ${ }^{67}$ Liposomes were also used to demonstrate that RNAi can be used successfully in vivo to significantly reduce functional expression of the $\mathrm{V}_{2} \mathrm{R}$ in the mouse kidney. ${ }^{68}$ Liposomes have been used in vivo in a number of studies in mouse models and may represent a potential method to deliver siRNA successfully. 

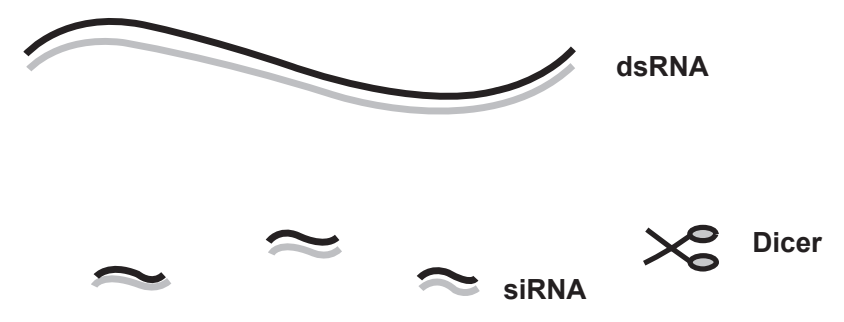

\section{o om}

siRNA unwinds and associates with RISC

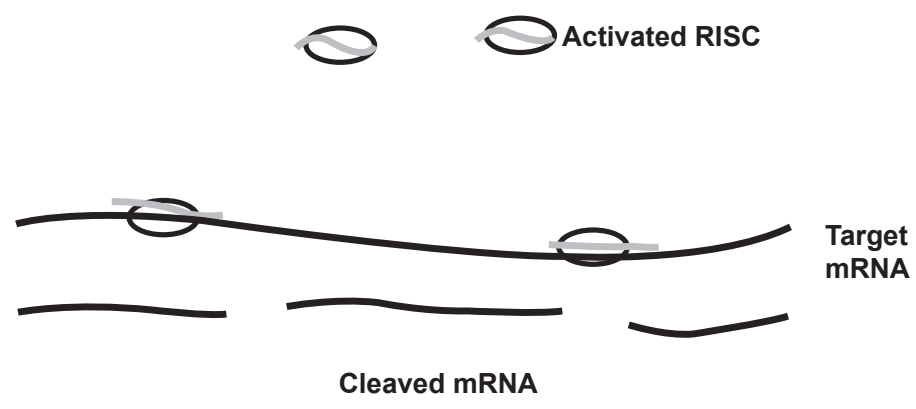

Cleavage occurs within siRNA sequence

Figure I Mechanism of RNA interference. dsRNA are processed by Dicer to produce siRNA of 2 I-23 nucleotides in length. The dsRNA unwinds, allowing one strand to bind to the RNA-induced silencing complex (RISC). Binding of the antisense RNA strand activates the RISC to cleave mRNAs containing a homologous sequence.

The delivery in vivo of siRNAs by cationic lipids has been described. Other strategies for local or systemic siRNA administration have been explored including chemical modifications of siRNA molecules, electropulsation, polyamine or other basic complexes, atelocollagen, virosomes and certain protein preparations.

In addition it is also possible to target siRNAs to specific organs including the liver. This can prevent unwanted effects on normal host tissues. Targeted delivery can be achieved by local administration or by injection via catheterization of the vascular supply to particular organs. Chemically modified siRNA could possibly also be covalently linked to ligands for cell-surface receptors, enabling delivery only to cells bearing these receptors ${ }^{66}$ The delivery of siRNA into model mammalian organisms has been achieved by intravenous injection or by electroporation of synthetic siRNAs directly into target tissues and organs..$^{69} \mathrm{In}$ rodents the rapid infusion of siRNA by hydrodynamic injection has seen successful transgene delivery in the liver with expression found in up to $40 \%$ of hepatocytes. ${ }^{70}$

Cruz and colleagues reported successful knockdown of A1AT in cell lines using A1AT siRNAs. ${ }^{71}$ They also successfully knocked down the expression of ZA1AT in ZA1AT overexpressing transgenic mice.

Pulmonary disease in A1AT deficiency is due to low serum levels of A1AT. Knockdown of ZA1AT expression would be of no benefit here, as it would further reduce the serum levels of circulating A1AT. As a gene therapy alternative to augmentations therapy the normal A1AT gene has been successfully introduced into the striated muscle cells of animals using an $\mathrm{AAV}$ vector both in vitro and in vivo. ${ }^{72,73} \mathrm{~A} 1 \mathrm{AT}$ gene replacement therapy may be of benefit to the pulmonary disease as the A1AT administered is in the form of MA1AT and trials are ongoing. ZA1AT knockdown and MA1AT replacement together could potentially be a very effective way of treating A1AT deficiency in PiZZ individuals. The major challenges to this therapy will include targeted delivery and chronicity.

\section{Peptide nucleic acids}

Although peptide nucleic acids were originally conceived as agents for double-stranded DNA binding, they were first exploited for gene therapy drug design. PNAs were first described by Nielson et al in $1991 .^{74}$ They are synthetic DNA 


\section{Anti-gene inhibition}

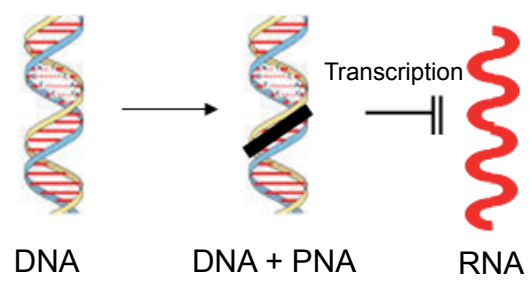

Anti-sense inhibition

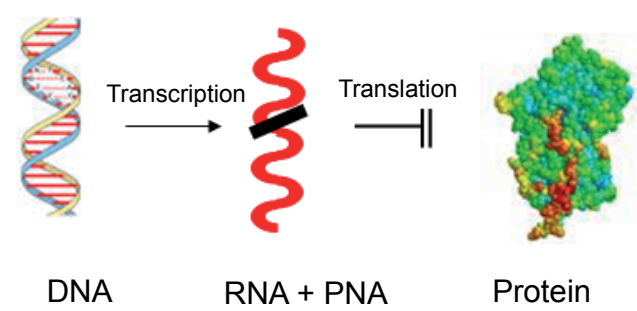

Figure 2 PNA-induced gene inhibition. PNA can bind with DNA leading to anti-gene inhibition, or it can bind with RNA leading to antisence inhition.

analogs in which the phosphodiester backbone has been replaced by repetitive units of the pseudo-peptide polymer $\mathrm{N}$-(2-aminoethyl) glycine to which the purine and pyrimidine bases are attached by a methyl carbonyl linker. ${ }^{75}$ PNAs hybridize to complementary DNA or RNA in a sequencedependent manner, according to the Watson-Crick hydrogen bonding scheme. In contrast to DNA, PNA can bind in either a parallel or antiparallel manner. ${ }^{76}$ The inhibition of gene expression by PNA is known as anti-gene inhibition when it occurs as a result of PNA binding to DNA, and as antisense inhibition when it occurs as a result of PNA binding to RNA. The synthetic uncharged peptide backbone provides PNA with unique hybridization characteristics. There is no electrostatic repulsion when PNAs hybridize to their target nucleic acid sequence and this gives a higher stability to the PNA-DNA or PNA-RNA duplexes than the natural homo- or heteroduplexes. ${ }^{77}$ PNAs are also not degraded by nucleases or proteases and therefore, cannot be directly used as primers, or be copied. ${ }^{75}$

PNAs can form strand invasion complexes or result in strand displacement in DNA and thus lead to the inhibition of gene transcription. Several in vitro studies have shown that the binding of PNA or bis-PNA to complementary DNA can efficiently block transcriptional elongation and inhibit the binding of transcriptional factors.

The interaction of PNAs with mRNA is independent of the RNA secondary structure. The antisense effects of PNAs occur through the steric interference of RNA processing or transport into the cytoplasm or through the interference of translation. This interference is caused by the binding of PNA to the mRNA. The antisense inhibition caused by siRNAs is due to RNase mediated degradation, however, PNAs are not substrates for RNase. ${ }^{78}$

PNAs could constitute highly efficient compounds for gene therapy as the oligomers have good stability and a strong binding affinity for DNA and RNA. They are also nontoxic, even at relatively high concentrations. However, as with siRNA, progress in the use of PNAs as tools for regulating gene expression slows at the in vivo stage. This is due to the slow uptake of naked PNA by living cells. Modifications of PNAs have led to significant improvements in the uptake of PNA in eukaryotic cells. PNAs can be coupled to DNA oligomers, to receptor ligands or more efficiently to peptides such as cell penetrating peptides which are rapidly internalized by mammalian cells. ${ }^{79}$ Such coupling markedly improves the uptake of PNAs by cells. As with siRNA liposomes can be of use to improve the uptake of PNAs when PNAs are incorporated into the liposomes. ${ }^{80}$

\section{Ribozymes}

Another method of inhibiting the expression of a gene is through the use of ribozymes. Ribozymes are naturally occurring catalytic RNA molecules that cleave RNA with high specificity. They behave like enzymes though there is no protein involved. The catalytic properties of RNA were discovered in the 1980s by Thomas Cech and Sidney Altman and the term 'ribozyme' was coined in $1982 .{ }^{81}$

Many ribozymes have either a hammerhead or hairpin shaped active center. Synthetically designed and specifically targeted hammerhead and hairpin ribozymes have been shown to be promising gene-targeting reagents. These ribozymes contain both a catalytic RNA domain that cleaves the target mRNA and a substrate-binding domain with a sequence antisense to the target mRNA sequence. Therefore, these gene-targeting ribozymes bind to the mRNA sequence through Watson-Crick interactions between the target sequence and the antisense sequence in the substrate-binding domain of the ribozyme. ${ }^{82}$

Ribozymes bind to their targets with a much higher specificity than siRNAs, which can potentially produce offtarget effects. There is no risk of an interferon response with ribozymes, unlike with siRNA. As with siRNA and PNAs there are problems using ribozymes to knockdown gene expression in vivo. The activity in vivo is not usually high enough to achieve the desired effects.

Human hepatoma cells were transduced with ribozymes to inhibit the expression of the mutant A1AT gene and express 
Ribozyme containing complementary sequence to target mRNA

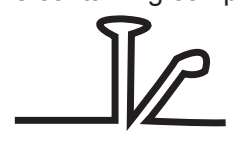

Target mRNA

Ribozyme binds to target mRNA

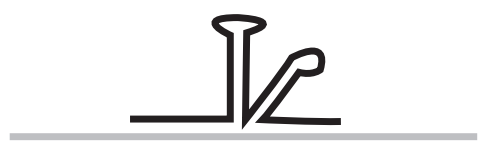

Cleavage

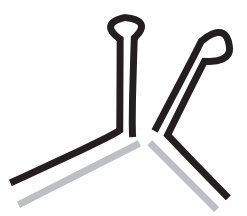

Figure 3 Mechanism of action of ribozymes. Ribozymes with a section of sequence complementary to the target mRNA will bind the mRNA and cleave it.

a modified A1AT gene. This ribozyme-mediated gene therapy did show inhibition of the mutant gene and expression of the modified A1AT gene; however the efficiency of this method is low. ${ }^{83}$

\section{Perspective}

Gene therapy has been under investigation since the emergence of restriction enzymes. Retroviral vectors were investigated and the first human trials began in the late $1980 \mathrm{~s} .{ }^{84}$ Gene therapy with recombinant adenovirus (rAd) has since developed and recombinant adenovirus (rAAV) trials were carried out in cystic fibrosis (CF) patients in $1993 .{ }^{85}$ rAAV trials were initiated in CF patients in $1995 .{ }^{86}$ Lentivirus and recombinant herpesvirus vectors have also been used. rAd, rAAV, lentivirus and recombinant herpesvirus vectors are capable of gene transfer and expression in vivo in nondividing cells. ${ }^{87}$ Nonviral gene transfer methods include DNA conjugates, and naked DNA injection and these came into use in gene therapy in the 1990s.

There are drawbacks to all of these methods of gene therapy. Wild-type Ad triggers innate and acquired immune responses. Recombinant Ad also triggers these responses in a dose-dependent manner. Gamma-retrovirus vectors have caused leukemia-like syndromes when used on bone-marrow stem cells. Recombinant AAV vectors can generate adaptive immune responses to the retained viral capsid proteins. Aerosol administration of nonviral vectors has been associated with an influenza-like syndrome and the efficacy of these systems in vivo is low and transient.

The only indications of clinically significant efficacy with gene therapy have been with onco-retrovirus vectors and a first-generation rAd vector, but these systems are associated with important limiting toxicities.

Gene therapy targeted to the liver has also been attempted. A study into the effectiveness of adenoviral delivery of interferon alpha to rats that were induced to develop human hepatocellular carcinoma and liver cirrhosis found that targeting of IFN-alpha expression to the liver significantly reduced liver tumor volume and ameliorated liver cirrhosis. ${ }^{88}$ In addition pseudotyped AAV vectors for the liver-specific expression of human blood coagulation factor IX (hFIX) have been investigated and expression in immunocompetent mice ranged from $36 \%$ to more than $2000 \%$ of normal. Expression was dose- and time-dependent. ${ }^{89}$ With respect to gene-targeted therapeutics for liver disease in general the efficacy of novel strategies still needs to be further validated in animal studies and confirmed in clinical trials. Particularly important are their efficient and safe delivery to the target tissue and the limitation of unintended harmful nonspecific effects such as transient or sustained inflammation.

siRNA, PNA and Ribozymes represent potential gene knockdown methods and with the ongoing investigation into their administration in vivo remains only a matter of time until a valid gene therapy emerges. For A1AT-deficient individuals this development would be most welcome. The liver disease caused by the polymerization of ZA1AT and the inflammatory effects due to the chemotactic characteristics of polymerized ZA1AT in the lung could both potentially be ameliorated by the knockdown of ZA1AT gene and protein expression in these organs. With the continuing investigations into A1AT augmentation therapy the adverse effects of A1AT deficiency may soon be completely overcome.

\section{Disclosure}

The authors report no conflicts of interest in this work.

\section{References}

1. Rogers J, Kalsheker N, Wallis S, et al. The isolation of a clone for human alpha 1-antitrypsin and the detection of alpha 1-antitrypsin in mRNA from liver and leukocytes. Biochem Biophys Res Commun. 1983;116:375-382.

2. Molmenti EP, Perlmutter DH, Rubin DC. Cell-specific expression of alpha 1-antitrypsin in human intestinal epithelium. $J$ Clin Invest. 1993;92:2022-2034. 
3. Hu C, Perlmutter DH. Cell-specific involvement of HNF-1beta in alpha(1)-antitrypsin gene expression in human respiratory epithelial cells. Am J Physiol Lung Cell Mol Physiol. 2002;282:L757-L765.

4. Travis J, Owen M, George P, et al. Isolation and properties of recombinant DNA produced variants of human alpha1-proteinase inhibitor. J Biol Chem. 1985;260:4384-4389.

5. Crystal RG, Brantly ML, Hubbard RC, Curiel DT, States DJ, Holmes MD. The alpha1-antitrypsin gene and its mutations. Chest. 1989;95:196-208.

6. Petrache I, Fijalkowska I, Zhen L, et al. A novel antiapoptotic role for alpha1-antitrypsin in the prevention of pulmonary emphysema. Am J Respir Crit Care Med. 2006;173:1222-1228.

7. Nita IM, Serapinas D, Janciauskiene SM. Alpha1-antitrypsin regulates CD14 expression and soluble CD14 levels in human monocytes in vitro. Int J Biochem Cell Biol. 2007;39:1165-1176.

8. Carrell RW, Lomas DA. Alpha1-antitrypsin deficiency - A model for conformational diseases. N Engl J Med. 2002;364:45-53.

9. Potempa J, Korzus E, Travis J. The serpin superfamily of proteinase inhibitors: structure, function, and regulation. J Biol Chem. 1994;269:15957-15960.

10. Coakley RJ, Taggart C, O’Neill S, McElvaney NG. Alpha1-antitrypsin deficiency: biological answers to clinical questions. Am J Med Sci. 2001;321:33-41.

11. Darlington GJ, Astrin KH, Muirhead SP, Desnick RJ, Smith M. Assignment of human alpha-1 antitrypsin to chromosome 14 by somatic cell hybrid analysis. Proc Natl Acad Sci U S A. 1982;79:870-873.

12. Schroeder WT, Miller MF, Woo SLC, Saunders GF. Chromosomal localization of the human alpha1-antitrypsin gene (PI) to 14q31-32. Am J Hum Genet. 1985;37:868-872.

13. Kok K, Wahab P, Houwen RHJ, et al. Heterozygous alpha- 1 antitrypsin deficiency as a co-factor in the development of chronic liver disease: a review. Neth J Med. 2007;65:160-166.

14. Greene CM, Miller SDW, Carroll T, et al. Alpha-1 antitrypsin deficiency: a conformational disease associated with lung and liver manifestations. J Inherit Metab Dis. 2008;31:21-34.

15. Seixas S, Garcia O, Trovoada MJ, Santos MT, Amorim A, Rocha J. Patterns of haplotype diversity within the serpin gene cluster at $14 \mathrm{q} 32.1$ : insights into the natural history of the alpha1-antitrypsin polymorphism. Hum Genet. 2001;108:20-30.

16. de Serres FJ. Worldwide racial and ethnic distribution of alpha1antitrypsin deficiency. Chest. 2002;122:1818-1829.

17. Tan JJ, Cutiongco-dela Paz EM, Avila JMC, Gregorio GV. Low Incidence of alpha1-antitrypsin deficiency among Filipinos with neonatal cholestatis. J Paediatr Child Health. 2006;42:694-697.

18. Khanna R, Alam S, Sherwani R, Arora S, Arora NK, Malik A. Alpha-1 antitrypsin deficiency among Indian children with liver disorders. Indian J Gastroenterol. 2007;25:191-193.

19. Seyama K, Nukiwa T, Takabe K, Takahashi H, Miyake K, Kira S. Siiyama (Serine 53 (TCC) to phenylalanine 53 (TTC)) a new alpha1antitrypsin-deficient variant with mutation on a predicted conserved residue of the serpin backbone. J Biol Chem. 1991;266:12627-12632.

20. Lomas DA, Evans DL, Stone SR, Chang WS, Carrell RW. Effect of the $\mathrm{Z}$ mutation on the physical and inhibitory properties of alpha1antitrypsin. Biochem. 1993;32:500-508.

21. Lomas DA, Evans DL, Finch JT, Carrell RW. The mechanism of Z alpha1-antitrypsin accumulation in the liver. Nature. 1992;357: 605-609.

22. Bathurst I, Travis J, George PM, Carrell RW. Structural and functional characterization of the abnormal $\mathrm{Z}$ alpha1-antitrypsin isolated from human liver. FEBS Lett. 1984;177:179-183.

23. Burrows JA, Willis LK, Perlmutter DH. Chemical chaperones mediate increased secretion of mutant alpha 1-antitrypsin (alpha 1-AT) Z: A potential pharmacological strategy for prevention of liver injury and emphysema in alpha 1-AT deficiency. Proc Natl Acad Sci US A. 2000;97:1796-1801.

24. Massi G. Pathogensis and pathology of liver disease associated with alpha1-antitrypsin deficiency. Chest. 1996;110:251S-255S.
25. Perlmutter DH. Liver injury in alpha1-antitrypsin deficiency: an aggregated protein induces mitochondrial injury. J Clin Invest. 2002;110:1579-1583.

26. Carrell RW. Alpha 1-Antitrypsin: molecular pathology, leukocytes, and tissue damage. J Clin Invest. 1986;78:1427-1431.

27. Lomas DA. Antitrypsin deficiency, the serpinopathies, and chronic obstructive pulmonary disease. Proc Am Thorac Soc. 2006;3:499-502.

28. Lin L, Schmidt B, Teckman JH, Perlmutter DH. A naturally occurring nonpolymeric mutant of alpha1-antitrypsin characterised by prolonged retention in the endoplasmic reticulum. J Biol Chem. 2001: 276:33893-33898.

29. Laurell CB, Eriksson S. [Hypo-alpha-1-antitrypsinemia.]. Verh Dtsch Ges Inn Med. 1964;70:537-539.

30. Taggart C, Cervantes-Laurean D, Kim G, et al. Oxidation of either methionine 351 or methionine 358 in alpha 1-antitrypsin causes loss of anti-neutrophil elastase activity. J Biol Chem. 2000;275:27258-27265.

31. Mulgrew AT, Taggart CC, Lawless MW, et al. Z $\alpha_{1}$-antitrypsin polymerizes in the lung and acts as a neutrophil chemoattractant. Chest. 2004;125(5):1952-1957.

32. Mahadeva R, Atkinson C, Li Z, et al. Polymers of $Z$ alpha1-antitrypsin co-localize with neutrophils in emphysematous alveoli and are chemotactic in vivo. Am J Pathobiol. 2005;166:377-387.

33. Woolhouse I, Bayley D, Stockley RA. Sputum chemotactic activity in chronic obstructive pulmonary disease: effect of alpha1-antitrypsin deficiency and the role of leukotriene B4 and interleukin 8. Thorax. 2002;57:709-714.

34. Hubbard RC, Fells G, Gadek J, Pacholok S, Humes J, Crystal RG. Neutrophil accumulation in the lung in alpha 1-antitrypsin deficiency. Spontaneous release of leukotriene B4 by alveolar macrophages. J Clin Invest. 1991;88:891-897.

35. Sharp HL, Bridges RA, Krivit W, Freier EF. Cirrhosis associated with alpha-1-antitrypsin deficiency: a previously unrecognized inherited disorder. J Lab Clin Med. 1969;73:934-939.

36. Zhou H, Ortiz-Pallardó ME, Ko Y, Fischer EP. Is heterozygous alpha-1-antitrypsin deficiency type PIZ a risk factor for primary liver carcinoma? Cancer. 2000;88:2668-2676.

37. Regev A, Guaqueta C, Molina EG, et al. Does the heterozygous state of alpha-1 antitrypsin deficiency have a role in chronic liver diseases? Interim results of a large case-control study. J Pediatr Gastroenterol Nutr. 2006;43:S30-S35.

38. Sveger T. Liver disease in alpha1-antitrypsin deficiency detected by screening of 200,000 infants. N Engl J Med. 1976;294:1316-1321.

39. McCracken AA, Karpichev IV, Ernaga JE, Werner ED, Dillin AG, Courchesne WE. Yeast mutants deficient in ER-associated degradation of the Z variant of alpha-1-protease inhibitor. Genetics. 1996;144: $1355-1362$.

40. Teckman JH, Burrows J, Hidvegi T, Schmidt B, Hale P, Perlmutter $\mathrm{DH}$. The proteasome participates in degradation of mutant alpha-1 antitrypsin $\mathrm{Z}$ in the endoplasmic reticulum of hepatoma-derived hepatocytes J Biol Chem. 2001;276:44865-44872.

41. Teckman JH, Perlmutter DH. Retention of mutant alpha-1 antitrypsin Z in the endoplasmic reticulum is associated with an autophagic response. Am J Physiol Gastrointest Liver Physiol. 2000;279:G961-G974.

42. Lawless MW, Mankan AK, Gray SG, Norris S. Endoplasmic reticulum stress - a double edged sword for Z alpha-1 antitrypsin deficiency hepatoxicity. Int J Biochem Cell Biol. 2008;40:1403-1414.

43. Lawless MW, Mankan AK, White M, O'Dwyer MJ, Norris S. Expression of hereditary hemochromatosis C282Y HFE protein in HEK293 cells activates specific endoplasmic reticulum stress responses. BMC Cell Biol. 2007;8:30.

44. Rakela J, Goldschmiedt M, Ludwig J. Late manifestation of chronic liver disease in adults with alpha-1-antitrypsin deficiency. Dig Dis Sci. 1987;32:1358-1362.

45. Wu Y, Whitman I, Momentum E, Moore K, Hippenmeyer P, Perlmutter DH. A lag in intracellular degradation of mutant alpha-1 antitrypsin correlates with the liver disease phenotype in homozygous PiZZ alpha-1 antitrypsin deficiency. Proc Natl Acad Sci US A. 1994;91:9014-9018. 
46. Carlson JA, Barton Rogers B, Sifers RN, et al. Accumulation of PiZ alpha1-antitrypsin causes liver damage in transgenic mice. JClin Invest. 1989;83:1183-1190.

47. Bowlus CL, Willner I, Zern MA, et al. Factors associated with advanced liver disease in adults with alpha1-antitrypsin deficiency. Clin Gastroenterol Hepatol. 2005;3:390-396.

48. Propst T, Propst A, Dietze O, Judmaier G, Braunsteiner H, Vogel W. High prevalence of viral infection in adults with homozygous and heterozygous alpha 1-antitrypsin deficiency and chronic liver disease. Ann Intern Med. 1992;117:641-645.

49. Eigenbrodt ML, McCashland TM, Dy RM, Clark J, Galati J. Heterozygous alpha 1-antitrypsin phenotypes in patients with end stage liver disease. Am J Gastroenterol. 1997;92:602-607.

50. Elzouki AN, Verbaan H, Lindgren S, Widell A, Carlson JA, Eriksson S. Serine protease inhibitors in patients with chronic viral hepatitis. JHepatol. 1997;27:42-48.

51. Scott BB, Egner W. Does alpha1-antitrypsin phenotype PiMZ increase the risk of fibrosis in liver disease due to hepatitis $\mathrm{C}$ virus infection. Eur J Gastroenterol Hepatol. 2006;18:521-523.

52. Rudnick DA, Shikspwashya O, Blomenkamp K, Teckman JH. Indomethacin increases liver damage in a murine model of liver injury from alpha1-antitrypsin deficiency. Hepatology. 2006;44:976-982.

53. Mencin A, Seki E, Osawa Y, et al. Alpha-1 antitrypsin Z protein (PiZ) increases hepatic fibrosis in a murine model of cholestasis. Hepatology. 2007;46:1443-1452.

54. Owen DR, Sivakumar R, Suh E-S, Seevaratnam M. Alpha-1-antitrypin deficiency resulting in a hitherto unseen presentation of hepatocellular carcinoma: polycythemia but with normal alpha fetoprotein. World $J$ Gastroenterol. 2006;12:4906-4907.

55. Sokol RJ. Fat-soluble vitamins and their importance in patients with cholestatic liver diseases. Gastroenterol Clin North Am. 1994;23:673-705.

56. Kemmer N, Kaiser T, Zacharias V, Neff GW. Alpha-1 antitrypsin deficiency: outcomes after liver transplantation. Transplant Proc. 2008;40:1492-1494.

57. McNab GL, Ahmad A, Mistry D, Stockley RA. Modification of gene expression and increase in alpha1-antitrypsin secretion after homologous recombination in alpha1-antitrypsin deficient monocytes. Hum Gene Ther. 2007;18:1171-1177.

58. Napoli C, Lemieux C, Jorgensen R. Introduction of a chimeric chalcone synthase gene into petunia results in reversible co-suppression of homologous genes in trans. Plant Cell. 1990;2:279-289.

59. Elbashir SM, Martinez J, Patkaniowska A, Lendeckel W, Tuschl T. Functional anatomy of siRNAs for mediating efficient RNAi in Drosophila melanogaster embryo lysate. EMBO J. 2001;20:6877-6888.

60. Brantl S. Antisense-RNA regulation and RNA interference. Biochim Biophys Acta. 2002;1575:15-25.

61. Hammond SM, Bernstein E, Beach D, Hannon GJ. An RNA-directed nuclease mediates post-transcriptional gene silencing in Drosophila cells. Nature. 2000;404:293-296.

62. Robertson HD, Webster RE, Zinder ND. Purification and properties of ribonuclease III from Escherichia coli. J Biol Chem. 1968;243:82-91.

63. Bernstein E, Caudy AA, Hammond SM. Role for a bidentate ribonuclease in the initiation step of RNA interference. Nature. 2001;409:363-366.

64. Omi K, Tokunaga K, Hohjoh H. Long-lasting RNAi activity in mammalian neurons. FEBS Lett. 2004;558:89-95.

65. Leung RK, Whittaker PA. RNA interference: from gene silencing to gene-specific therapeutics. Pharmacol Ther. 2005;107:222-239.

66. Lieberman J, Song E, Lee S-K, Shankar P. Interfering with disease: opportunities and roadblocks to harnessing RNA interference. Trends Mol Med. 2003;9:397-404.

67. Yano J, Hirabayashi K, Nakagawa S, et al. Antitumor activity of small interfering RNA/cationic liposome complex in mouse models of cancer. Clin Cancer Res. 2004;10:7721-7726.
68. Hassan A, Tian Y, Zheng W, Ji H, Sandberg K, Verbalis JG. Small interfering RNA-mediated functional silencing of vasopressin V2 receptors in the mouse kidney. Physiol Genomics. 2005;21:382-388.

69. Dillon CP, Sandy P, Nencioni A, Kissler S, Rubinson DA, Van Parijs L. RNAi as an experimental and therapeutic tool to study and regulate physiological and disease processes. Annu Rev Physiol. 2005;67:147-173.

70. Liu F, Song Y, Liu D. Hydrodynamics-based transfection in animals by systemic administration of plasmid DNA. Gene Ther. 1999;6:1258-1266.

71. Cruz PE, Mueller C, Cossette TL, et al. In vivo post-transcriptional gene silencing of alpha- 1 antitrypsin by adeno-associated virus vectors expressing siRNA. Lab Invest. 2007;87:893-902.

72. Song S, Morgan M, Ellis T, et al. Sustained secretion of human alpha1-antitrypsin from murine muscle transduced with adeno-associated virus vectors. Proc Natl Acad Sci U S A. 1998;95:14384-14388.

73. Flotte TR. Recombinant adeno-associated virus gene therapy for cystic fibrosis and alpha-1 antitrypsin deficiency. Chest. 2002;121:98-121.

74. Nielsen PE, Egholm M, Berg RH, Buchardt O. Sequence-selective recognition of DNA by strand displacement with a thymine-substituted polyamide. Science. 1991;254:1497-1500.

75. Pellestor F, Paulasova P. The peptide nucleic acids (PNAs), powerful tools for molecular genetics and cytogenetics. Eur J Hum Genet. 2004; 12:694-700.

76. Rasmussen H, Kastrup JS, Nielsen JN, Nielsen JM, Nielsen PE. Crystal structure of a peptide nucleic acid (PNA) duplex at $1.7 \mathrm{~A}$ resolution. Nat Struct Biol. 1997;4:98-101.

77. Jensen KK, Orum H, Nielsen PE, Norden B. Hybridization kinetics of peptide nucleic acids (PNA) with DNA and RNA studied with BIAcore technique. Biochemistry. 1997;36:5072-5077.

78. Knudsen H, Nielsen PE. Antisense properties of duplex- and triplexforming PNAs. Nucleic Acids Res. 1996;24:494-500.

79. Cutrona G, Carpaneto EM, Ulivi M, et al. Effects in live cells of a c-myc anti-gene PNA linked to a nuclear localization signal. Nat Biotechnol. 2000;18:300-303.

80. Nastruzzi C, Cortesi R, Esposito E, et al. Liposomes as carriers for DNA-PNA hybrids. J Control Release. 2000;68:237-249.

81. Kruger K, Grabowski PJ, Zaug AJ, Sands J, Gottschling DE, Cech TR. Self-splicing RNA: autoexcision and autocyclization of the ribosomal RNA intervening sequence of Tetrahymena. Cell. 1982;31:147-157.

82. Trang P, Kim K, Liu F. Developing RNase P ribozymes for genetargeting and antiviral therapy. Cell Microbiol. 2004;6:499-508.

83. Ozaki I, Zern MA, Liu S, Wei DL, Pomerantz RJ, Duan L. Ribozymemediated specific gene replacement of the alpha1-antitrypsin gene in human hepatoma cells. J Hepatol. 1999;31:53-60.

84. Rosenberg SA, Aebersold P, Cornetta K, et al. Gene transfer into humans-immunotherapy of patients with advanced melanoma, using tumor-infiltrating lymphocytes modified by retroviral gene transduction. N Engl J Med. 1990;323:570-578.

85. Crystal RG, McElvaney NG, Rosenfeld MA, et al. Administration of an adenovirus containing the human CFTR cDNA to the respiratory tract of individuals with cystic fibrosis. Nat Genet. 1994;8:42-51.

86. Flotte TR, Carter B, Conrad C, et al. A phase I study of an adenoassociated virus-CFTR gene vector in adult $\mathrm{CF}$ patients with mild lung disease. Hum Gene Ther. 1996;7:1145-1159.

87. Flotte TR. Gene therapy: the first two decades and the current stateof-the-art. J Cell Physiol. 2007;213:301-305.

88. Huang KW, Huang YC, Tai KF, Chen BH, Lee PH, Hwang LH. Dual therapeutic effects of interferon-alpha gene therapy in a rat hepatocellular carcinoma model with liver cirrhosis. Mol Ther. 2008;16:1681-1687.

89. Grimm D, Zhou S, Nakai H, et al. Preclinical in vivo evaluation of pseudotyped adeno-associated virus vectors for liver gene therapy. Blood. 2003;102:2412-2419. 
